\title{
Mapping Grain Morphology and Grain Orientations by Laboratory Diffraction Contrast Tomography
}

\author{
Jun Sun $^{1 *}$, Hrishikesh Bale ${ }^{2}$, Florian Bachmann ${ }^{1}$, Jette Oddershede ${ }^{1}$, Stephen T. Kelly ${ }^{2}$, William Harris ${ }^{2}$ \\ and Erik Lauridsen ${ }^{1}$ \\ 1. Xnovo Technology, Køge, Denmark. \\ 2. Carl Zeiss X-ray Microscopy, Pleasanton, CA, USA. \\ * Corresponding author: jsun@xnovotech.com
}

Crystallographic imaging (i.e. imaging of crystallites/grains in polycrystalline materials) is primarily known from electron microscopy, and particularly the introduction of the electron back-scattering diffraction (EBSD) technique in the early 1990's, has made it a routine tool for research and/or development related to metallurgy, functional ceramics, semi-conductors, geology etc. The ability to image the grain structure in such materials is instrumental for understanding and optimization of material properties and processing. However, the destructive nature of 3D EBSD prevents the technique from directly evaluating the microstructure evolution when subject to either mechanical, thermal or other environmental conditions.

Non-destructive x-ray-based crystallographic imaging methods allow for extended '4D' time dependent studies but have to date been primarily the domain of a limited number of synchrotron facilities $[1,2]$. However, recently laboratory-based diffraction contrast tomography was introduced as an additional imaging modality on a commercially available X-ray microscope and thereby opened up a whole new range of possibilities for studies on the effect of $3 \mathrm{D}$ crystallography on materials performance.

Laboratory diffraction contrast tomography (LabDCT) [3-5] makes use of high-resolution diffraction images acquired on a ZEISS Xradia Versa X-ray microscope. Figure 1 schematically illustrates the working principles of LabDCT. The instrument uses a polychromatic, divergent X-ray beam, instead of the parallel monochromatic beam typically used in the synchrotron X-ray DCT technique. An aperture is placed between the source and the sample, to constrain the incoming X-ray beam to illuminate the sample only in the center region of the detector. For the DCT scan, a beam-stop is used to block the transmitted X-rays to increase the sensitivity of the diffraction signals. A high-resolution detector is placed at the Laue focal plane with equal source-detector distance. By this mean, the crystal grains fulfilling the Bragg condition focus the divergent X-ray beams into a line in the diffraction pattern.

Recent developments of the LabDCT technique have extended its capabilities to include full reconstruction of 3D grain structure including both grain morphology and crystallographic orientation, thereby making the LabDCT more comparable to conventional 3D-EBSD data - while still supporting 4D time dependent studies. With both the grain morphology and orientation, it is possible to extract the full five parameters describing the grain boundary characteristics, which, opens new possibility for statistical studies of grain boundary properties. The unique non-destructive 3D crystallographic imaging capabilities of LabDCT complement the structural data obtained by traditional absorption-based tomography and together they provide an unprecedented insight into the structure of materials.

We will present a selection of LabDCT applications (see Figure 2 for one example) with particular emphasis on its non-destructive operation, demonstrated through correlative analysis as well as 4D 
evolutionary studies obtained by repeating the imaging procedure numerous times on the same sample. We will discuss the boundary conditions of the current implementation, point to the future of the technique and discuss ways in which this can be correlatively coupled to related techniques for a better understanding of materials structure evolution in 3D.

\section{References:}

[1] HF Poulsen et al., MRS Bull. 29(3) (2004), p. 166.

[2] W Ludwig et al., J. Appl. Crystallogr. 41(2) (2013), p. 310.

[3] SA McDonald et al., Sci Reports 5 (2015), p. 14665.

[4] C Holzner et al., Micros. Today 24(4) (2016), p. 34.

[5] F Bachmann et al., '3D Grain Reconstruction from Laboratory Diffraction Contrast Tomography', submitted.

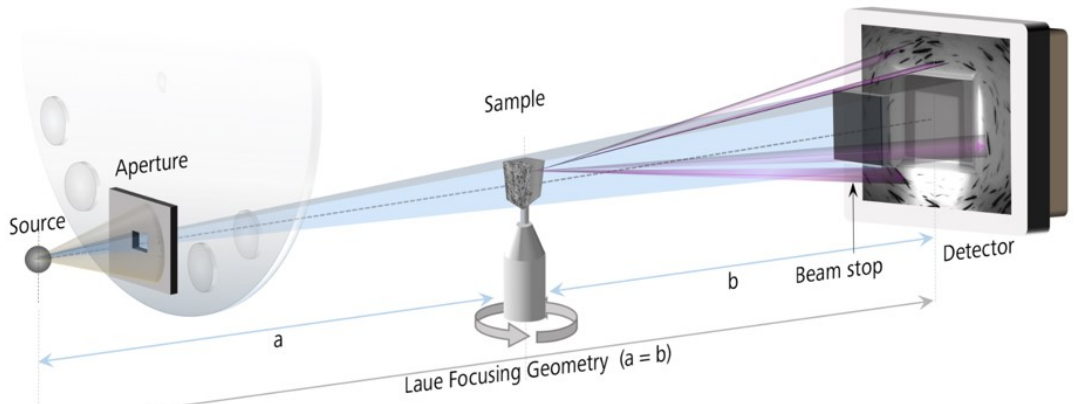

Figure 1. Schematic showing the experimental setup of LabDCT in the laboratory X-ray microscope.

(a)

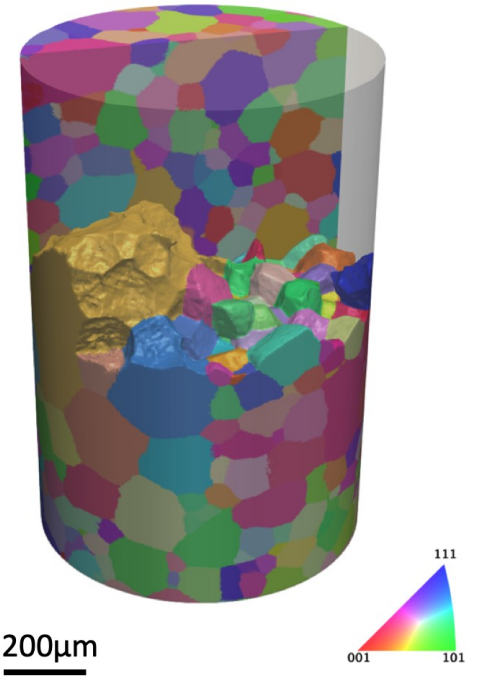

(b)

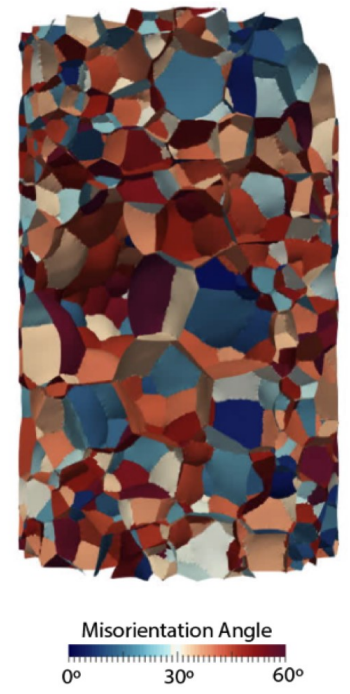

Figure 2. (a) 3D rendering of an iron sample characterized by LabDCT, with IPF coloring. (b) $3 \mathrm{D}$ rendering of the grain boundaries in the same sample volume, colored by the misorientation angles. 